\section{IMPACTS OF SAFE COMMUNITY PROGRAMS IN JAPANESE COMMUNITIES}

Yoko Shiraishi. Japan Institution for Safe Communities, Japan

\subsection{6/injuryprev-2016-042156.324}

Background In Japan, the movement of community safety promotion called "Safe Community (SC)" was firstly launched by a community in 2006 and it was designated in 2008. Since then, the movement has been gradually expanding across the country. As of March 2016, there are 13 designated communities in Japan and another few more are working on safety promotion based on the SC morel.

Although the "designation" as members of the Safe Community network is a good way of branding the communities, the communities are more interested in the impacts of SC in various aspects such as improvement of safety and accompanying changes in the communities. This study therefore aims to provide a broad-ranging analysis of the impacts of the SC programs at the municipality level.

Methods The annual reports submitted by the designated Japanese communities were examined to see changes in the structure, mechanism, resources of safety promotion at the community level, outputs of programs and impacts such as the mortality from external causes. Based on the information from the written materials, semi-structured interview were conducted to the relevant parties such as politicians, city government, citizens and so on.

Results In all communities working on the community safety promotion with the SC model, the structures of cross sectoral collaboration and the cycle of the program operation as Plan-DoCheck-Act was developed along with their situations. In addition, the citizens' involvement in the movements has been promoted. Those improvements have also caused changes in outputs. As a result of a sequence of those changes, some impacts have been observed in mortality and morbidity from some external causes such as traffic accidents, suicide, falling and so on. In addition, the newly established surveillance system related to the medical data have made it possible to see the impacts on the medical cost to those injuries.

Conclusions Although comprehensiveness and multiplicity of the movements are features of the SC, these aspects have made it difficult to see the clear impacts of the SC programs. Those difficulties can drive politicians cautious about application of the SC model into their community governance. Currently, the improvements in the infrastructure and outcomes have been already evaluated in many communities but it is still difficult to identify the outcomes. Therefore, the evaluation schemes in relation to the medical cost which are now under the development in some communities will shed light on the impacts evaluation in other communities. Once the firm assessment system is developed and become made good use at the community level, decision makers and practitioners can see how their efforts change their communities and it will contribute to the dynamic community involvement.

\section{EVALUATING COMPLEX COMMUNITY-BASED VIOLENCE AND INJURY PREVENTION INTERVENTIONS: A STATISTICAL FRAMEWORK}

${ }^{1}$ Shrikant I Bangdiwala, ${ }^{2}$ Yoko Shiraishi. 'University of North Carolina Injury Prevention Research Centre, Chapel Hill, NC, USA; ${ }^{2}$ Japan Institute for Safe Communities, Osaka, Japan

\subsection{6/injuryprev-2016-042156.325}

Background Violence and injury prevention interventions conducted in community settings are complex due to multiple reasons, including: (1) they address a complex situation by addressing many risk factors simultaneously, (2) they are often overlapped in their implementation, (3) they are implemented in phases over time, (4) implementation must be consistent with community priorities and budgetary limitations, and (5) they are not conducted in randomised, controlled studies. Thus, evaluating their impact is not statistically straightforward.

Methods A methodological analytic framework is proposed that uses random effects meta-regression methodology and incorporates a taxonomy that allows for complex interventions to be 'disentangled' into their active components. The framework is illustrated with pilot study data from communities in Japan participating in the Safe Communities global effort, using motor vehicle related deaths and hospitalizations as the indicators of effectiveness.

Results Information from 8 communities in Japan for 20082011 (4 years each) provided information. Being designated implied a reduction of 1.6/100,000 MV related deaths annually, and that being designated implied a statistically significant reduction in 81/100,000 MV related hospitalizations! We also found that media campaigns were somewhat effective in explaining some of the reduction in hospitalizations in these communities in Japan, but that education was not.

Conclusions The proposed statistical framework is very useful to understand the effectiveness of community-based, multi-component, dynamic interventions. The framework may help researchers and policy-makers evaluating the effectiveness and impact of complex intervention programs.

\section{HEALTHY PUBLIC SPACE AS BASIC PRINCIPLE FOR WALKABILITY IN MEXICO CITY}

Perla Castañeda. Universidad Autónoma Metropolitana, Mexico City

\subsection{6/injuryprev-2016-042156.326}

Background Car reliance is the most costly transport mode because of its negative externalities. The city administration in Mexico City (CDMX) has tried to solve traffic by increasing roads to prioritise motorised vehicles, thus, inducing traffic. Only $24 \%$ population drives cars, the rest walk, cycle or use public transport. Hence, there is an urgent appeal and grants for walkability and making infrastructure improvements to protect pedestrians to reduce risk of road traffic injuries.

Methods Public space has been studied from different theoretical perspectives. Revised literature has not related Health Promotion to public space. The epistemological and pragmatic approaches of public space have led to reach the materiality of pedestrian infrastructure as healthy public space.

Results Since 2009, I targeted efforts to produce healthy public space by detecting a case study aimed to regenerate a long sidewalk, with 45 years of decay that runs along an urban high-way. This potential footpath belongs to the second most populated borough in CDMX with 1.2 million, but mostly is the daily 